\title{
Estudio de costo del tratamiento de la aspergilosis invasora en pacientes oncológicos pediátricos. Santiago. Chile
}

\author{
Claudia Moreno M., Gladys del Valle M. y Paulina Coria D. la H.
}

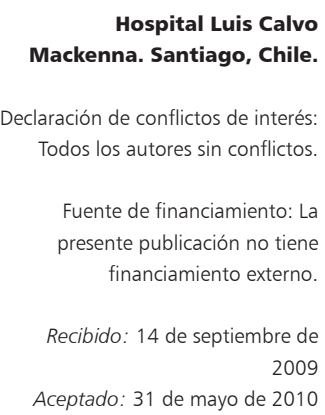

Correspondencia a:

Claudia Moreno Moreno clau.moreno.m@gmail.com

\section{Economical evaluation of the treatment of invasive aspergillosis in pediatric oncology patients. Santiago. Chile}

Introduction: Invasive aspergillosis (IA) is a serious opportunistic infection in immunocompromised patients. Transplant recipients and patients with cancer represent the highest risk group. The antifungal treatment involves prolonged hospitalization and high economic resources. Objective: to estimate costs represented by IA as an intercurrent complication of oncologic treatment. Patients and Method: Retrospective case-control study. Estimation of the cost of treatment in pediatric oncologic patients with IA in the Hospital Luis Calvo Mackenna during the years 2007-2008 was done. A control for each case of IA paired by sex, age, number of diagnosis and clinical department was selected. Results: There were 13 patients during the observation period. The attributable cost of treatment of aspergillosis was US \$23,600 and the cost for each indicator was: hospital days US \$ 16,500; antifungal therapy US \$ 7,000; and serum galactomannan US \$ 100. Discussion: In this study, the cost of treating IA is mainly due to hospitalization and antifungal medications. Three patients acquired IA in spite of staying in a protected environment.

Key words: Aspergilosis, cost, resources, children.

Palabras clave: Aspergilosis, costo, recursos, pediátrico.

\section{Introducción}

L a aspergilosis invasora (AI) es una causa poco frecuente de infección en pacientes inmunocompetentes; sin embargo, en pacientes inmunocomprometidos es la infección fúngica filamentosa más frecuente, causando importante morbimortalidad. Representa $85 \%$ de las infecciones fúngicas invasoras causadas por hongos filamentosos y constituye la principal causa de mortalidad por infección pulmonar en pacientes sometidos a trasplantes de precursores hematopoyéticos (TPH). La tasa de mortalidad en pacientes receptores de trasplante con diagnóstico de AI fluctúa entre 74 y $92 \%{ }^{1}$. Asociado a esto, los pacientes presentan estadía hospitalaria prolongada y costosos tratamientos médicos, por lo que los centros de salud deben invertir importantes recursos económicos ${ }^{2}$.

El género Aspergillus se encuentra ampliamente distribuido en la naturaleza asociado a zonas húmedas o materia orgánica en descomposición y es considerado una de las especies más ubicuas debido a sus requisitos metabólicos simples y su gran capacidad de soportar un amplio rango de temperaturas ${ }^{3}$. Estos hongos producen alta cantidad de conidias que están adaptados para la diseminación aérea. La inhalación de conidias de Aspergillus por el ser humano no suele producir manifestaciones clínicas, salvo reacciones alérgicas como rinitis alérgica, sinusitis alérgica, aspergilosis broncopulmonar alérgica y alveolitis alérgica extrínseca. Sin embargo, en pacientes inmunocomprometidos la inhalación de esporas fúngicas alcanza el tracto respiratorio inferior produciendo aspergilosis pulmonar o aspergiloma. Desde el tracto respiratorio puede diseminarse por vía hematógena hasta manifestarse como $\mathrm{AI}^{4}$. Aspergillus fumigatus es la especie aislada con mayor frecuencia, cercano a $90 \%$ de los casos. Otras especies aisladas son A. flavus, A.niger, A. terreus y A. nidulans ${ }^{5}$.

Durante los últimos 10 años ha aumentado la incidencia de pacientes inmunocomprometidos, principalmente por aumento de los trasplantes a nivel mundial. Esto genera gran riesgo de desarrollar AI en los pacientes receptores del trasplante, representando el grupo de mayor riesgo ${ }^{6}$. En el mundo se ha reportado infección por Aspergillus entre 2 y $26 \%$ en pacientes con TPH y entre 1 y $15 \%$ en pacientes con trasplante de órgano sólido.

Según consensos internacionales (EORTC/MSG) ${ }^{4}$ la AI ha sido clasificada en tres categorías según la probabilidad diagnóstica: probada, probable y posible. La AI probada requiere una muestra histopatológica que documente la infección y un cultivo positivo para Aspergillus en muestras de sitios estériles. La definición de AI probable debe cumplir criterios en tres categorías: factores de riesgo del paciente (inmunocompromiso), manifestaciones clínicas (síntomas, signos y hallazgos radiológicos) y evidencia microbiológica (cultivo positi- 
vo para Aspergillus, galactomanano positivo o $\beta$-glucano positivo) y AI posible si falta alguno de los factores presentes en AI probable ${ }^{7}$ (Tabla 1).

Los factores de riesgo del paciente para presentar AI son neutropenia prolongada; TPH, especialmente alogénicos; trasplante de órgano sólido, principalmente durante el primer año del transplante; SIDA (recuento de CD4 < 100 céls $/ \mathrm{mm}^{3}$ ) e inmunodeficiencias primarias como la enfermedad granulomatosa crónica ${ }^{2}$.

Una publicación chilena de 1988 descrita por los autores Oddó y Acuña ${ }^{8}$ estudió 5.612 autopsias entre los años 1960-1986. Se encontraron 175 agentes infecciosos oportunistas en los tejidos de 151 individuos. El 75\% de estos oportunistas correspondió a agentes micóticos. El 23,4\% (41 casos) fueron originados por Aspergillus, describiéndose una letalidad de $88 \%$ por este hongo; el pulmón fue el órgano más frecuentemente comprometido. Las enfermedades linfohematológicas fueron la condición predisponerte más frecuente (linfomas, leucemias, agranulocitosis, mielomas, mielofibrosis, histiocitosis maligna). El promedio de edad de los sujetos estudiados fue de 65 años (26 semanas-84 años) y para el caso específico de aspergilosis fue de 57 años. En este reporte se describe un aumento de los casos de infecciones oportunistas a partir de la década del 80, en relación al período previo.

Los factores ambientales y nosocomiales también participan en el desarrollo de aspergilosis. El aire interior nosocomial contiene 1 a 10 ufc de Aspergillus por $\mathrm{mm}^{3}$, cuyas esporas miden entre 2,5 a 3,5 $\mu \mathrm{m}$ de diámetro y pueden mantenerse en suspensión en el aire. Ciertas condiciones ambientales como construcciones o remodelaciones, sistemas de ventilación o de aire acondicionado, sistemas de calefacción defectuosos o contaminados, aislamientos contra el fuego y movimientos de polvo sobre techos falsos pueden incrementar el número de esporas en el aire ambiental nosocomial ${ }^{9}$. Estos factores se han asociado con la aparición de brotes de aspergilosis nosocomial que pueden estudiarse con métodos de tipificación genotípicas, identificación de las fuentes de infección e instauración de medidas eficaces de control ${ }^{10}$.

El tratamiento antifúngico de elección en la AI es la monoterapia con voriconazol y, en forma alternativa, se puede administrar anfotericina $\mathrm{B}$, caspofungina o posaconazol $^{2}$. Estos medicamentos tienen costos bastante elevados, a los que se agrega la estadía hospitalaria prolongada, mayor número de interconsultas médicas y exámenes de laboratorio, lo que lleva a invertir importantes recursos económicos.

Entre las medidas para prevenir la aspergilosis nosocomial se encuentran la disminución del número de esporas del ambiente en contacto con el paciente susceptible. Esto se logra mediante combinación de filtros de aire de alta eficiencia (HEPA), sistemas de recambio de aire, salas con presión positiva y control aéreo en general. Estas
Tabla 1. Clasificación de enfermedad fúngica invasora (AI) en pacientes inmunocomprometidos con cáncer de acuerdo a los criterios EORTC/MSG ${ }^{4}$

\begin{tabular}{|c|c|}
\hline Categoría & Definición \\
\hline Al probada o definida & $\begin{array}{l}\text { - Observación y/o aislamiento de hongos en examen microscópico } \\
\text { directo o cultivo a partir de muestra clínica representativa de un sitio } \\
\text { estéril o hemocultivo } \\
\text { - Histopatología compatible }\end{array}$ \\
\hline Al probable & $\begin{array}{l}\text { - Si existen factores predisponentes en el hospedero } \\
\text { - Elementos clínico-radiológicos sugerentes } \\
\text { - Un estudio micológico positivo, pero no concluyente: galactomanano } \\
\text { o } \beta \text {-glucano positivo o cultivo positivo de sitio no estéril }\end{array}$ \\
\hline Al posible & - Cuando falta alguno de los factores presentes en probable Al \\
\hline
\end{tabular}

medidas requieren una inversión económica inicial pero pueden disminuir los costos a largo plazo ${ }^{11}$.

Hasta el momento no existen en Chile estudios de costos de AI en pacientes pediátricos. Por este motivo se realizó el análisis de costo comparativo de niños con diagnóstico de aspergilosis durante los años 2007 y 2008 utilizando tres indicadores de costo: estadía hospitalaria, tratamiento farmacológico y estudio de galactomanano. El objetivo de este estudio es analizar el costo de estos indicadores en el tratamiento de la aspergilosis invasora en unidades oncológicas pediátricas.

\section{Pacientes y Método}

Diseño del estudio. Estudio descriptivo, retrospectivo, caso-control. Se utilizó el protocolo de la infección intrahospitalaria de la $\mathrm{OMS}^{12}$ para determinar el costo asociado al tratamiento de AI en pacientes pediátricos oncológicos del Hospital Luis Calvo Mackenna durante los años 2007 y 2008, desde el 17 de enero del 2007 hasta el 26 de diciembre del 2008. Revisión retrospectiva de los registros clínicos de pacientes con $\mathrm{AI}$, seleccionando un control por cada caso de AI de acuerdo a las características: sexo, edad, número de diagnósticos y servicio clínico.

Casos y controles permanecieron en ambiente protegido sólo en el período de la intervención del TPH. Las hospitalizaciones posteriores se realizaron en otras unidades del hospital, sin esta condición ambiental.

\section{Población de estudio}

Casos. Se incluyeron pacientes pediátricos bajo 18 años de edad (2-17 años) con diagnóstico de enfermedad oncológica y sospecha clínica de AI. Los pacientes fueron estudiados con cultivos microbiológicos e imágenes de TAC: de tórax, senos paranasales, cerebro y ecocardiografía, según el contexto clínico individual. Para realizar el diagnóstico de AI se utilizaron los criterios definidos por la European Organization for Research and Treatment 
of Cancer/Mycoses Study Group (EORTC/MSG) ${ }^{4-7}$, que incluye casos de AI posible, probable y probada (Tabla 1). Se excluyeron casos de aspergilosis alérgicas o localizadas en pacientes inmunocompetentes.

Controles. Los controles fueron seleccionados durante el mismo período que los casos de AI. Se identificó un control por cada caso según las características clínicas del caso: edad ( \pm 12 meses), sexo, diagnóstico oncológico principal y número de diagnósticos $( \pm 1)$ y servicio clínico.

Indicadores de costo. Se utilizaron sólo tres indicadores de costo del tratamiento de AI: días de hospitalización; tratamiento antifúngico y galactomanano sérico como examen de laboratorio, considerando dos determinaciones en días separados para hacer el diagnóstico. No se incluyeron procedimientos endoscópicos de lavado broncoalveolar ni biopsia pulmonar, así como tampoco exámenes radiológicos, debido a la dificultad de la estimación económica real de cada examen. Los valores económicos fueron establecidos según los costos del arancel FONASA.

Tabla 2. Características clínicas de los casos de aspergilosis invasora y los controles

\begin{tabular}{|c|c|c|}
\hline Característica & $\begin{array}{l}\text { Casos } \\
\text { (n 13) }\end{array}$ & $\begin{array}{l}\text { Controles } \\
\text { (n 13) }\end{array}$ \\
\hline Promedio de edad en años (rango) & $10,5(2-17)$ & $10,75(3-18)$ \\
\hline Sexo masculino & $7(53,8 \%)$ & $7(53,8 \%)$ \\
\hline \multicolumn{3}{|l|}{ Diagnóstico } \\
\hline Leucemia linfática aguda (LLA) & $7(53,8 \%)$ & $9(69,2 \%)$ \\
\hline Leucemia mieloide aguda (LMA) & $3(21,4 \%)$ & $1(7,1 \%)$ \\
\hline Leucemia mieloide crónica (LMC) & $1(7,1 \%)$ & $1(7,1 \%)$ \\
\hline Osteosarcoma & $1(7,1 \%)$ & $1(7,1 \%)$ \\
\hline Aplasia medular & $1(7,1 \%)$ & Sin casos \\
\hline Retinoblastoma & Sin casos & $1(7,1 \%)$ \\
\hline Antecedente de TPH & $7(53,8 \%)$ & $7(53,8 \%)$ \\
\hline Aspergilosis invasora posible & $2(15,3 \%)$ & Sin aspergilosis \\
\hline Aspergilosis invasora probable & $4(30,7 \%)$ & Sin aspergilosis \\
\hline Aspergilosis invasora probada o demostrada & $7(53,8 \%)$ & Sin aspergilosis \\
\hline \multicolumn{3}{|l|}{ Servicio clínico } \\
\hline Oncología / trasplante de médula ósea & 11 & 13 \\
\hline Pensionado & 1 & 0 \\
\hline Segunda infancia & 1 & 0 \\
\hline Ambiente protegido & 3 & 8 \\
\hline Mortalidad & $4(30,7 \%)$ & $0(0 \%)$ \\
\hline Promedio de estadía hospitalaria & 66,2 & 31,5 \\
\hline $\begin{array}{l}\text { Promedio de estadía hospitalaria en unidades } \\
\text { de paciente crítico }\end{array}$ & 29,5 & 25,5 \\
\hline
\end{tabular}

\section{Análisis de Resultados}

Estimación del costo atribuible al tratamiento de AI. Se calculó la diferencia de los costos económicos del tratamiento de aspergilosis en relación a los controles considerando la duración de estadía hospitalaria, el costo del tratamiento farmacológico antifúngico y del estudio diagnóstico con galactomanano sérico (Platelia ${ }^{\mathrm{TM}}$ Aspergillus EIA-Bio-Rad, MARNES-La Coquette-France).

Análisis estadístico. Los análisis se realizaron mediante pruebas no paramétricas de Sign Rank test-Wilcoxon o Mann-Whitney Sum rank test, debido a los resultados encontrados con muestras pequeñas. Los cálculos consideran significación estadística con un valor de p menor a 0,05 .

\section{Resultados}

Se observaron 13 pacientes con diagnóstico de AI durante el período 2007-2008, 7 casos el año 2007 y 6 casos el 2008. Dos casos correspondieron a AI posible, 4 casos a AI probable y 7 casos a AI probada o demostrada. Las características clínicas de los casos de AI fueron: promedio de edad de 10,5 años, 7 casos $(53,8 \%)$ de sexo masculino. Siete casos $(53,8 \%)$ tuvieron antecedente de $\mathrm{TPH}$, tres de los cuales presentaron AI durante la hospitalización en que se realizó el TPH en unidad con ambiente protegido. Los sujetos controles tuvieron un promedio de edad de 10,7 años, 7 (53,8\%) fueron de sexo masculino, 7 pacientes $(53,8 \%)$ tenían antecedente de TPH, 8 de ellos con trasplante en curso durante su hospitalización permaneciendo en unidades con ambiente protegido. Las características clínicas de casos de AI y controles se muestran en la Tabla 2.

Los casos de AI y los pacientes controles presentan similitud en las características clínicas en $97,7 \%$ para la edad, 100\% para sexo, 93\% para el número de diagnósticos y $83,3 \%$ para el servicio clínico al cual pertenecían (Tabla 3), lo que permite comparar ambos grupos y estimar el costo del tratamiento de $\mathrm{AI}$ en pacientes pediátricos oncológicos.

La mortalidad en el grupo de pacientes con AI fue de $30,7 \%$, dos pacientes fallecieron el año 2007 y dos el año 2008 hasta la fecha del estudio (enero 2009). La mortalidad fue atribuible a aspergilosis invasora en tres casos. No se registra mortalidad en el grupo control sin AI.

El promedio de días de hospitalización para el grupo de pacientes con AI fue de 66,2 días (9-175 días) y para el grupo control fue de 31,5 días (6-74 días), encontrando una diferencia promedio de 34,7 días mayor para el grupo de pacientes con AI. Al evaluar la estadía en unidades de paciente crítico (UPC), el promedio de días para los casos de AI fue de 29,5 días (0-99 días) y 25,5 días (0-94 días) para los controles, mostrando 4 días extra en promedio 
Tabla 3. Calidad de pareo entre los casos de aspergilosis y los controles

\begin{tabular}{|lcccc|}
\hline Variable & Casos & Controles & Porcentaje de pareo & Valor $\mathbf{p}^{*}$ \\
\hline Edad (años) & 10,5 & 10,7 & $97,7 \%$ & NS \\
\hline Sexo masculino & $53,8 \%$ & $53,8 \%$ & $100 \%$ & NS \\
\hline Número de diagnósticos & 5,14 & 4,78 & $93 \%$ & NS \\
\hline Pacientes en un mismo servicio clínico & 12 & 14 & $83,3 \%$ & NS \\
\hline NS, no significativo * Cálculo estadístico utilizando la prueba de Wilcoxon & & \\
\hline
\end{tabular}

Tabla 4. Costo atribuible del tratamiento de aspergilosis invasora en pacientes pediátricos oncológicos

\begin{tabular}{|c|c|c|c|c|}
\hline Indicador de costo & Casos & Controles & \multicolumn{2}{|c|}{$\begin{array}{l}\text { Exceso de costo atribuible del } \\
\text { tratamiento de Al durante el 2007-2008 }\end{array}$} \\
\hline Costo promedio de días de hospitalización & $\$ 14.090 .082,14$ & $\$ 4.818 .039,07$ & $\$ 9.272 .043$ & (US $\$ 16.500)$ \\
\hline Costo promedio del tratamiento antifúngico ${ }^{1}$ & $\$ 3.953 .000$ & 0 & $\$ 3.953 .000$ & (US $\$ 7.000)$ \\
\hline Costo promedio galactomanano sérico ${ }^{2}$ & $\$ 72.178,57$ & $\$ 23.035,71$ & $\$ 49.143$ & (US $\$ 100)$ \\
\hline Total & $\$ 18.115 .260,71$ & $\$ 4.841 .074,78$ & $\$ 13.274 .186$ & (US \$23.600) \\
\hline
\end{tabular}

para los casos de AI. Tres casos de AI desarrollaron la infección durante la hospitalización en unidades de trasplante con ambiente protegido.

Todos los pacientes recibieron voriconazol endovenoso, siete pacientes recibieron además voriconazol oral. Cuatro pacientes recibieron caspofungina, cinco anfotericina B deoxicolato y sólo un paciente recibió anidulafungina. Tres pacientes recibieron tres o más antifúngicos.

\section{Análisis de costo}

Estimación del costo atribuible del tratamiento de AI. Se estimó el costo atribuible del tratamiento de AI en relación a los controles considerando los costos netos sin IVA que cancela el Hospital, encontrándose un costo atribuible promedio total para cada caso de $\$ 13.274 .185$ pesos chilenos (US \$23.600). Cabe destacar que este costo no incluye los exámenes radiológicos ni los procedimientos debido a la subestimación del costo real encontrado en los registros del hospital.

El costo atribuible promedio por cada indicador de costo fue: días de hospitalización: \$9.272.043 pesos (USD \$16.500) costo de medicamentos antifúngicos: \$3.953.000 pesos (USD \$7.000); costo de galactomanano sérico: \$49.142 pesos (USD \$100) tal como se observa en la Tabla 4.
Análisis estadístico. No se encontraron diferencias estadísticamente significativa entre el grupo de casos y de controles para la edad, el sexo, el número de diagnósticos y el servicio clínico de estadía, por lo que fue posible realizar la comparación del costo entre ambos grupos.

Al analizar el costo del tratamiento de aspergilosis, incluyendo los tres indicadores de costo (días de hospitalización, medicamentos antifúngicos y estudio con galactomanano) se encontraron diferencias estadísticamente significativas $(\mathrm{p}<0,05)$.

\section{Discusión}

Los pacientes inmunocomprometidos han aumentado en Chile y el mundo principalmente por mayor incidencia de trasplantes y patología oncológica, lo que conlleva importantes riesgos de infecciones sistémicas. Una de las infecciones graves más frecuentes en pacientes susceptibles es la AI. Esta infección trae como consecuencia mayor estadía hospitalaria en unidades clínicas especiales, procedimientos invasores diagnósticos, medicamentos antifúngicos, elevada mortalidad y altos recursos económicos.

Durante los años de este estudio se encontraron 7 casos de AI el 2007 y 6 casos de AI el 2008 en pacientes 
pediátricos oncológicos del hospital, existiendo un aumento en la incidencia de pacientes con AI en relación a años anteriores. Es destacable que 53,8\% del total de pacientes con AI tenía como antecedente ser receptores de $\mathrm{TPH}$, representando el grupo de mayor riesgo, tal como se describe en la literatura médica. En cuanto al análisis del costo atribuible a AI se obtuvo en promedio para cada caso un valor cercano a los US \$23.600. En este estudio, el costo es atribuido principalmente a la estadía hospitalaria y los fármacos antifúngicos. Sin embargo, en este análisis no están considerados los costos de exámenes radiológicos ni procedimientos médicos, por lo que no podemos hacer comentarios en relación a esos valores. El costo de AI es significativo para el hospital si se considera el total de casos de AI por año, representando un valor cercano a los US\$165.000 para el 2007, año que encontramos el mayor número de pacientes en esta serie.

Este estudio presenta varias limitaciones entre las que se encuentran el desconocimiento de la incidencia de AI en el Hospital Luis Calvo Mackenna durante años previos a este estudio y la falta de precisión en la estimación de costo del tratamiento de AI debido a que no se incluyeron todas las variables involucradas en el costo total del tratamiento, entre ellos, los exámenes radiológicos y procedimientos diagnósticos como la biopsia pulmonar y lavado broncoalveolar. Esto se debe a la dificultad de la estimación económica real de cada indicador que es cancelada por el hospital. Estos indicadores omitidos afectan el resultado total del costo atribuible del tratamiento de AI, pudiendo existir mayor impacto en la diferencia de casos de AI y pacientes controles.

El ambiente hospitalario se relaciona con la transmisión de enfermedades infecciosas en pacientes receptores de TPH y con neutropenia prolongada, especialmente infecciones fúngicas filamentosas como Aspergillus spp y bacterianas como Legionella spp. En el caso de aspergilosis existe alta correlación entre las ufc $/ \mathrm{m}^{3}$ ambientales y la incidencia de brote de la enfermedad; no existe, sin embargo, consenso en el punto de corte más adecuado $\left(0,2-0,9 \mathrm{ufc} / \mathrm{m}^{3}\right)$. Lo demostrado hasta la fecha es que un valor menor a $0,009 \mathrm{ufc} / \mathrm{m}^{3}$ representa un factor protector para el desarrollo de $\mathrm{AI}^{13-14}$. En nuestro hospital no se estudia la presencia de hongos ambientales en forma rutinaria; ésta podría ser una medida a instaurarse para determinar el impacto clínico, tanto en el control ambiental como en la disminución del riesgo de infección en pacientes oncológicos pediátricos.

Con el objetivo de disminuir el número de conidias de Aspergillus en el aire ambiental y prevenir infecciones fúngicas en pacientes de riesgo se ha utilizado el ambiente protegido, definido como pieza individual con presión positiva que impide la entrada de microorganismos en suspensión aérea, flujo unidireccional, laminar, que evita el bloqueo al flujo de salida de aire, sistema de filtración de 99,97 - 99,99\% de eficiencia con filtros HEPA que remueve partículas mayores a 0,3 a 0,12 $\mu \mathrm{m}$ y, de manera opcional, recirculación permitida por flujo laminar. Los sistemas de control de aire incluyen mecanismos de control de la humedad, calefacción, enfriamiento y uso de ventiladores ${ }^{15}$. Los costos de mantención de filtros HEPA son altos y requieren un cambio al saturarse, con un año de duración, aproximadamente.

En nuestro hospital, junto con la creación del Programa de Trasplante de Precursores Hematopoyéticos, se implementó una unidad especial con sistema de ambiente protegido para pacientes sometidos a TPH que opera desde el año 1999, funcionando con un sistema adecuado de mantención de filtros y control de partículas en forma periódica. Otro grupo de riesgo preocupante son los pacientes oncológicos en tratamiento con quimioterapia ( $\sin \mathrm{TPH}), 46,2 \%$ del total, cuyo factor de riesgo más importante es la neutropenia profunda y prolongada, y que ni en nuestro hospital, y según nuestro conocimiento, en ningún otro hospital del sistema público de salud, se cuenta con ambiente protegido.

Los pacientes de alto riesgo de adquirir AI necesitan un control ambiental, siendo ideal la hospitalización en ambiente protegido; no obstante, encontramos en esta serie tres pacientes receptores de trasplante que pese a hospitalizarse en ambiente protegido, desarrollaron infección fúngica. Esto podría explicarse por su infección durante los traslados frecuentes de los pacientes a otras zonas del hospital que no cuentan con ambiente protegido. Por estos motivos, es fundamental contar con medidas preventivas como el uso de mascarillas de alta eficiencia (N95) cuando los pacientes transitan por diversas áreas del establecimiento, norma vigente en este hospital. Cada centro debe evaluar la factibilidad de la implementación del ambiente protegido, considerando las ventajas de sus propiedades y las limitaciones de sus recursos.

Agradecimientos. A Jorge Morales, químico-farmacéutico del Hospital Luis Calvo Mackenna, por su colaboración en los datos de los costos del tratamiento antifúngico de los pacientes. A Enrique Hernández, Escuela de Salud Pública, Facultad de Medicina, Universidad de Chile, por su colaboración en el análisis de significación estadística. A Cecilia Tapia, médico microbiólogo, ICBM, Universidad de Chile, por su participación en la corrección del documento.

\section{Resumen}

Introducción: La aspergilosis invasora (AI) es una infección oportunista grave en pacientes inmunocomprometidos. Pacientes receptores de transplantes y oncológicos representan el grupo de mayor riesgo. El tratamiento antifúngico involucra hospitalización prolongada y altos recursos económicos. Objetivo: Estimar los costos 
involucrados en el tratamiento de la AI como complicación intercurrente en pacientes con cáncer. Pacientes y Método: Estudio caso-control, retrospectivo. Estima el costo del tratamiento de AI en pacientes pediátricos oncológicos del Hospital Luis Calvo Mackenna durante los años 2007 y 2008. Resultados: Se incluyeron 13 pacientes con AI y sus respectivos 13 controles. El costo atribuible de la hospitalización en aquellos pacientes que cursaron con AI fue de US \$23.600. El costo atribuible para cada indicador fue: US \$16.500 para días de hospitalización; US $\$ 7.000$ para medicamentos antifúngicos y US $\$ 100$ para galactomanano sérico. Discusión: En este estudio, el costo del tratamiento de AI se debe principalmente a la estadía hospitalaria y fármacos antifúngicos. Encontramos tres pacientes que desarrollaron AI estando en ambiente protegido.

\section{Referencias}

1.- Segal B H, Walsh T J. Current approaches to diagnosis and treatment of invasive aspergillosis. Am J Respir Crit Care Med 2006; 173: 707-17.

2.- Walsh T J, Anaissie E J, Denning D W, Herbrecht R, Kontoyiannis D P, Marr K A, et al. Treatment of aspergillosis: clinical practice guidelines of the Infectious Diseases Society of America. Clin Infect Dis 2008; 46: 327-60.

3.- Barnes P D, Marr K A. Aspergillosis: spectrum of disease, diagnosis, and treatment. Infect Dis Clin North Am 2006; 20: 545-61.

4.- Ascioglu S, Rex J H, de Pauw B, Bennett J E, Bille J, Crokaert F, et al. Invasive Fungal Infections Cooperative Group of the European Organization for Research and Treatment of Cancer; Mycoses Study Group of the National Institute of Allergy and Infectious Diseases. Defining opportunistic invasive fungal infections in immunocompromised patients with cancer and hematopoietic stem cell transplants: an international consensus. Clin Infect Dis 2002; 34: $7-14$
5.- Perea S, Patterson T F. Invasive Aspergillus infections in hematologic malignancy patients. Semin Respir Infect. 2002; 17: 99-105.

6.- Singh N, Paterson DL. Aspergillus infections in transplant recipients. Clin Microbiol Rev 2005; 18: 44-69.

7.- De Pauw B, Walsh T J, Donnelly J P, Stevens D A, Edwards J E, Calandra T, et al. Revised Definitions of Invasive Fungal Disease from the European Organization for Research and Treatment of Cancer/Invasive Fungal Infections Cooperative Group and the National Institute of Allergy and Infectious Diseases Mycoses Study Group (EORTC/MSG) Consensus Group. Clin Infect Dis 2008; 46: 1813-21.

8.- Oddó D, Acuña G. Opportunistic infections in Chilean autopsy cases, 1960-1986. Bull Pan Am Health Organ 1988; 22 (1): 17-26.

9.- Pannuti C. Hospital environmental for high risk patients. In Wenzel R, editor: Prevention and Control of Nosocomial Infections. Ed3 ${ }^{\circ}, 1997$. Williams and Wilkins, C Baltimore, Maryland, E.U.A. 24, p 463-89.

10.- Marr K A, Carter R A, Boeckh M, Martin P, Corey L. Invasive aspergillosis in allogeneic stem cell transplant recipients: changes in epidemiology and risk factors. Blood 2002; 100: 4358-66.

11.- Versley D, Environmental Services, in Mayhall C G, editor: Hospital epidemiology and infection control, ed $2^{\circ}, 1999$. Williams and Wilkins C: Philadelphia, Pa, E.U.A. 69: 1047-53.

12. Protocolo para determinar el costo de la infección hospitalaria. OMS. www.paho.org/ Spanish/AD/DPC/CD/protocolo.pdf (Accedido: 7 enero 2009).

13. Rhame F S. Prevention of nosocomial aspergillosis. J Hosp Infect 1991; 18: 466-72.

14. Sherertz R J, Belani A, Kramer B S, Elfenbein G J, Weiner R S, Sullivan M L, et al. Impact of air filtration on nosocomial Aspergillus infections. Unique risk of bone marrow transplant recipients. Am J Med 1987; 83: 709-18.

15. Sehulster L, Chinn RY; CDC; HICPAC. Guidelines for environmental infection control in health-care facilities. Recommendations of CDC and the Healthcare Infection Control Practices Advisory Committee (HICPAC). MMWR Recomm Rep 2003; 52: 1-42. 